\title{
GPS ENABLED ANDROID APPLICATION FOR BUS SCHEDULE SYSTEM
}

\author{
Abhishek Dilip Bhonge ${ }^{1}$, Deepak Dattatray Kankhare ${ }^{2}$, Prasad Laxmanrao Takate ${ }^{3}$ \\ ${ }^{1,2,3}$ Student Department of Computer Engineering, GES R.H.S.COE, Maharashtra, India
}

\begin{abstract}
Nowadays in this fast life where everyone in is hurry to reach their destinations. Waiting for bus is a hectic and even many of us are unaware of the bus timings, hence to overcome this difficulty we have come up with the system of GPS Enabled Android Application For Bus Schedule System is considered in this study. The major feature included in the Android Application is user can know all the Nasik City Bus timings. This includes the various ways to find out the Bus available, by giving the appropriate bus number, by providing the source and destination or by providing the bus stop. Second module consisting of capturing the current latitude, longitude and location of the current position of the user using the GPS facility available in mobile Third module includes the facility of security call, if user feels unsecure or any disaster occur, just by pressing a single button user can notify to its closed ones with its current position. Then the Android and Java platforms are used to develop the application using eclipse tool.
\end{abstract}

Keywords: GPS, Google Android, Android SDK, Google API.

\section{INTRODUCTION}

Android is becoming very popular in embedded market for two mainstream reasons. First, source code is completely free; moreover there are no royalty fees for Java VM (Virtual Machine). Second deriving from the first, Android is highly suitable for expansion as the developer see fit. This paper presents an approach using Android SDK [1][2][3] and Google API [4][5][6] as to develop application for intelligent mobile devices, mainly GPS. The core part of this paper is as follows.

Bus schedule system is an application of Android phones. This application uses the GPS function also, available in most smart phones today, to pin point your location fairly accurately. With this application installed on your Smart phone, all you need to do is to start up your apps when you needed. User has to select the source and destination. This application will show you the bus timings with the accurate fare as per your source and destination. It also runs in the background so you are free to use your phone for the other activities like playing games, listening songs etc. This application also gives the users current position. And also includes the user's security.

\subsection{Project Scope}

This is purely android based application which only runs on android devices or android phones. Basically, this application provides all the city/town bus time table with and appropriate fare. Locating of user and security factors also considered in this. This application can also save the time of user that who wants to travel through the bus.

\subsection{User Classes and Characteristics}

- User must have GPS in his/her smart phone.

- The Application should not disturb user while performing any task on their handset. Like making or receiving calls, sending SMS, MMS and etc.

- When user enters the source and destination then application should show appropriate bus schedule.

- When user presses the security key it directly send the message to the registered people.

- If some area's time table changes then administrator is provided with a separate module to change or update the timetable as required which will be reflective to the users.

\subsection{Design and Implementation Constraint \\ - Android operating system device/phone. \\ - Internet Connection \\ - Phone having GPS facility.}

\section{SYSTEM/APPLICATION ARCHITECTURE}

This system acts as a bus timing consultant in which user is provided with required bus timings according to his need for a specific source. Apart from this, this system also provides facility for user to know his current position on map. As well as it also generates security SMS as when needed by the user. For the above system to provide the user with accurate results a proper input from the user is essential that is to get the proper timings of bus schedules user should provide proper input of his required source. If the user is new traveler in the city then he can know his current position in the city by using this 
application. Along with this he can also get the fare of the required route which he wishes to travel. For any user (local or remote) if there is a natural disaster or if he feels unsecured while travelling then he is also able to generate and send alerts SMSs to the needful people. To provide the system with the entire above functionality database is maintained at the server side. This database consists of proper schedule (time table) of the buses available at various locations which helps in getting the accurate information as required by the user. In this whole architecture the mobile available with the user acts as a client who connects to the server in which the database is maintained and thus obtains the required accurate information from the server by giving proper request to the server.

\section{IMPLEMENTATION}

Proposed system highlights on the bus schedule of the city. We can develop it for the various cities. This application considers the bus city timings with an appropriate fare. We can develop it same for the railway and air schedule also.

Proposed application consisting of the following modules-

- Bus schedule

- Obtaining user location

- Security call/alert SMS.

- Reset location

\subsection{Bus Schedule}

In this module, (i.e. nothing but checks availability in the Fig.3)When user presses the bus schedule button, a new window will open. The user has to select the source location from which he/she wants to start the journey and also has to select the destination to which he/she wants to reach. By selecting the particular source and destination the appropriate schedule is gets displayed with and fare. This schedule is taken from the database which is "MySQL" at the server side. The entire bus schedule with the fare is stored in it. By performing an appropriate functionality it will get the contents from the same. Separate server is provided to the city bus controller (MSRTC) through which they can access their database. If there are certain changes in the schedule of the buses or in fare, the server side will edit/insert/delete the operation and performs it. As soon as done with it, the user who uses this application automatically updates the software/application. There is no need to update the application as it is connected with the internet. That's the client/server communication done in this paper.

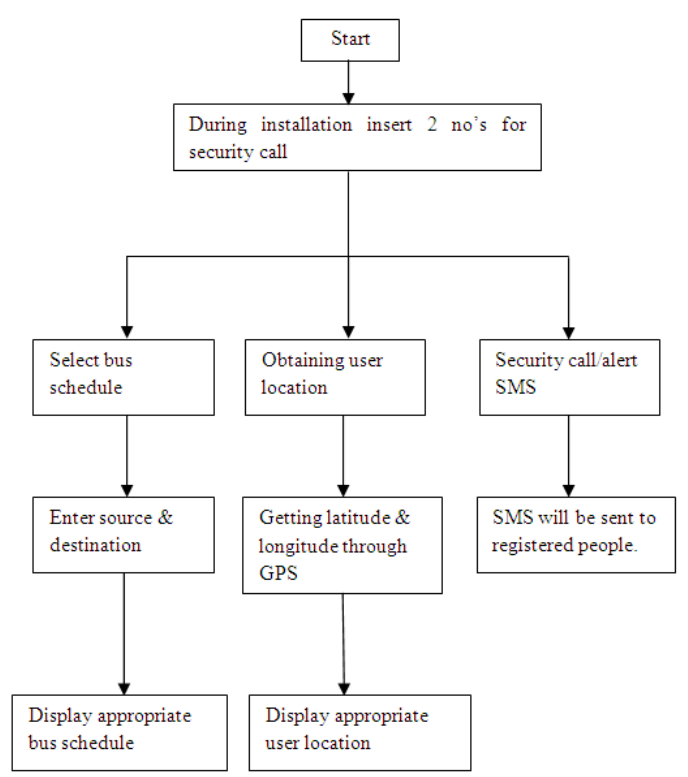

Fig-2 Flow of application

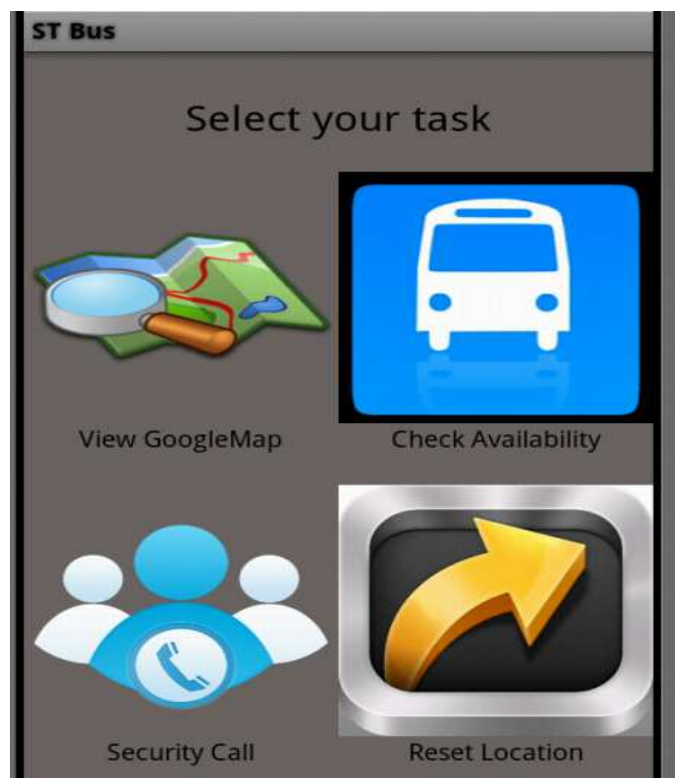

Fig -3: Layout of application

\subsection{Obtaining User Location}

In this module, (i.e. view Google map in Fig: 3) User command to obtain his current position by pressing the "View Google Map" button. As soon as the system gets the command from the user to obtain the current position Then system activates its GPS system and obtains the current latitude and longitude for user which is then used to display the current position of the user on Google map. (Please see fig.4) 


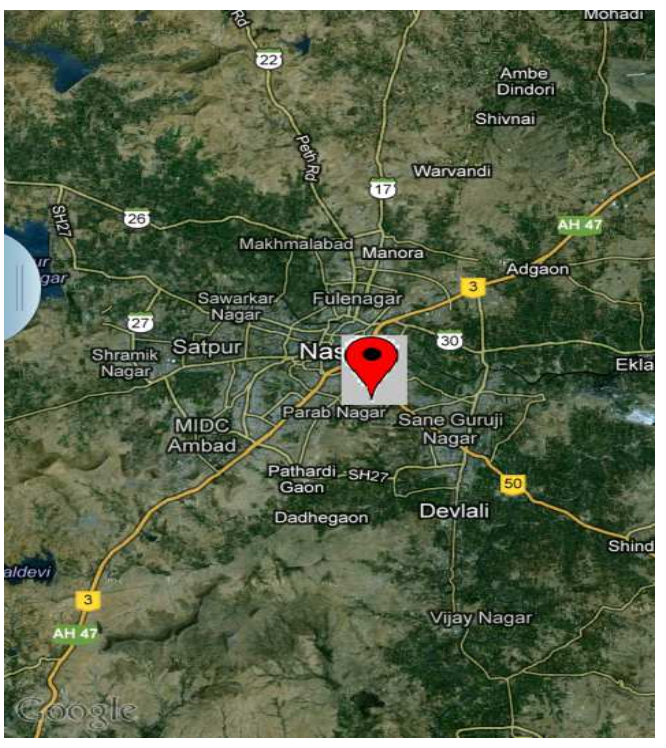

Fig- 4: Obtaining user location

\subsection{Security call/alert sms}

User command to generate alert SMS by pressing the Security Alert key (Fig-3 Security call button) As soon as the system gets the user command to generate alert SMS the system tracks the current position of user and converts it into its appropriate physical location and automatically sends SMS to the security squad or the needful people as provided by user.

\subsection{Reset Location}

When user first clicks on view Google map option then user gets his current location through GPS facility by getting latitude and longitude. This get current position is stored in the database. (i.e. SqlLite) By clicking this reset location option this stored location is gets erased.

\section{PROPOSED ENHANCEMENTS}

By fixing a GPS system in every bus we can track each and every bus from central location this reduces the traffic problems and leads to the better work. We can also have the RIFD signals within the bus so that, two consecutive buses can communicate. This leads to the available of seats within the bus. If the number of vacant seats is available then we will have red signal on the app else it will display the green signal within it.

\section{CONCLUSIONS}

Learning while developing was the main theme of this project. This project allows us to get acquainted with the work culture, people and environment. Project was great opportunity for us to learn and work in the environment.
Considering the feature of application such as bus schedule and security measures we hope that our application will play an important role.

\section{ACKNOWLEDGEMENTS}

We all marvel at the beautiful rose. The rose with all its beauty and grandeur. But seldom we pause and thank the gardener who patiently manure and watered it. The project we have made is the fruits of many such unseen hands. Before we get into thick of things, we would like to add a few heartfelt words for the people who were part of this project in numerous ways. People who gave unending support right from the stage, the project idea were conceived. We would like to take this opportunity to thank all those who have played an important role in the development of the project "GPS Enabled Android Application for Bus Schedule System". There are too many personalities in the endeavor who helped nonetheless; I want to thank a few people who are lining in the clouds for us.

This work was supported \& sponsored by "Maharashtra State Road Transport Cooperation (MSRTC)" Nasik Division (M.S. India). We are also thankful to the "Prof.N.V.Alone" for their extensive support.

\section{REFERENCES}

[1].Android SDK, http://developer.android.com/sdk/index.html [2]. Dong Shiwei, Wang Wei Xiang, Ting and Linming Zhang Hong-cai," Android 2 SDK introduction and application development" Sung gang Asset Management Corp. Limited 2010

[3]. She Zhilong, Yu-Hsun, Cheng Ming-jie, Chen Xiaofeng, Guo Zhijun, "Google Android SDK development, examples of documents" Delightpress 2010.

[4]. Google API, http://code.google.com/intl/zh-TW/apis/maps/ [5]. Editorial Board, "Google API Go to application design , development, examples" Sung Gang Asset Management Corp. Limited, 2010

[6]. Jiang W, and Gong Xiaopeng, "Detailed Google Map Google API developed two swords together with Google Earth wall”, Sung Gang Asset Management Corp. Limited, 2010

[7]. GOOGLE INC. (Hrsg.): Android Software Development Kit (SDK). Google Inc., http://developer.android.com/sdk/index.html. - Android 2.2, Release 2

[8]. “Android, http://www.android-app-market.com/androidarchitecture.html."

[9]. W.-M. Lee, Beginning Android 4 Application Development

[10]. J.-C. C. Y.-C. L. Jin-Tsong Jeng, Chen-Chia Chuang, "Systematic design for the global positional systems with application in intelligent Google android phone," 2011.

[11]. "Android, http://www.android.com/." 


\section{BIOGRAPHIES}

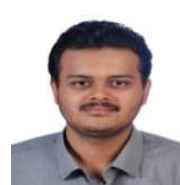

Abhishek D.Bhonge pursuing the Bachelor of Engineering in Computer at Gokhale Education Society's R.H.Sapat College of Engineering, Nasik, M.S. India.

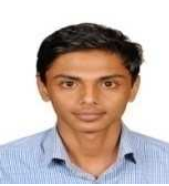

Deepak Kankhare pursuing the Bachelor of Engineering in Computer at Gokhale Education Society's R.H.Sapat College of Engineering, Nasik, M.S. India.

Prasad Takate pursuing the Bachelor of Engineering in Computer at Gokhale Education Society's R.H.Sapat College of Engineering, Nasik, M.S. India. 